\title{
Solute carrier transporters: potential targets for digestive system neoplasms
}

This article was published in the following Dove Press journal: Cancer Management and Research

\author{
Jing Xie ${ }^{1,2}$ \\ Xiao Yan Zhu',2 \\ Lu Ming Liu',2 \\ Zhi Qiang Meng',2 \\ 'Department of Integrative Oncology, \\ Fudan University Shanghai Cancer \\ Center, ${ }^{2}$ Department of Oncology, \\ Shanghai Medical College, Fudan \\ University, Shanghai 200032, People's \\ Republic of China
}

\begin{abstract}
Digestive system neoplasms are the leading causes of cancer-related death all over the world. Solute carrier (SLC) superfamily is composed of a series of transporters that are ubiquitously expressed in organs and tissues of digestive systems and mediate specific uptake of small molecule substrates in facilitative manner. Given the important role of SLC proteins in maintaining normal functions of digestive system, dysregulation of these protein in digestive system neoplasms may deliver biological and clinical significance that deserves systemic studies. In this review, we critically summarized the recent advances in understanding the role of SLC proteins in digestive system neoplasms. We highlighted that several SLC subfamilies, including metal ion transporters, transporters of glucose and other sugars, transporters of urea, neurotransmitters and biogenic amines, ammonium and choline, inorganic cation/anion transporters, transporters of nucleotide, amino acid and oligopeptide organic anion transporters, transporters of vitamins and cofactors and mitochondrial carrier, may play important roles in mediating the initiation, progression, metastasis, and chemoresistance of digestive system neoplasms. Proteins in these SLC subfamilies may also have diagnostic and prognostic values to particular cancer types. Differential expression of SLC proteins in tumors of digestive system was analyzed by extracting data from human cancer database, which revealed that the roles of SLC proteins may either be dependent on the substrates they transport or be tissue specific. In addition, small molecule modulators that pharmacologically regulate the functions of SLC proteins were discussed for their possible application in the treatment of digestive system neoplasms. This review highlighted the potential of SLC family proteins as drug target for the treatment of digestive system neoplasms.
\end{abstract}

Keywords: solute carrier, digestive system neoplasms, carcinogenesis, tumor progression, chemoresistance, small molecule modulators

\section{Introduction}

Neoplasms of digestive system, with varying locations in organs and tissues related to the gastrointestinal tract, remain to be the leading causes of cancer-related mortality all over the world. ${ }^{1}$ Composed of a lot of aggressive cancers, such as esophageal squamous-cell carcinoma, hepatocellular carcinoma, cholangiocarcinoma (CHOL), pancreatic adenocarcinoma, and colorectal carcinoma according to the classification of World Health Organization in $2010,{ }^{2}$ neoplasms of digestive system overall gain great difficulty in treatment and prognosis, in spite of rapid development of medical technologies. ${ }^{3}$ The aggressiveness of neoplasms of digestive system can be due to rapid mutation, proliferation, population expansion, invasion and metastasis, as well as high resistance to therapeutic agents. ${ }^{4}$
Correspondence: Zhi Qiang Meng Department of Integrative Oncology, Shanghai Cancer Center, Fudan University, 270 Dong'An Road, Shanghai 200032, People's Republic of China Email mengzhq@yeah.net 
Transporter proteins function to mediate the translocation of substrates through the biological membranes, either from extracellular toward intracellular region or vice versa. ${ }^{5}$ Two superfamilies of transporter proteins, ATP-binding cassette (ABC) and solute carrier (SLC), are globally expressed throughout tissues and organs of human body with different abundance. ${ }^{6}$ Different from ABC transporters that mediate energy-dependent efflux of intracellular substrates, ${ }^{7}$ SLC family proteins primarily carry out the uptake of small molecules. ${ }^{8}$ There are $\sim 400$ SLC proteins that are divided into 52 subfamilies based on their sequence similarity. ${ }^{9}$ A particular SLC subfamily owns high selectivity and can only interact with a narrow group of molecules as its substrates. ${ }^{10}$ Under physiological condition, some SLC family proteins exert important functions to maintain the homeostasis of human body, because SLC transporters facilitate the uptake of some life-essential molecules such as amino acid and glucose. ${ }^{11}$ SLC transporters can also regulate the absorption of metals, which often mediate the normal functions of important enzymes. ${ }^{12}$ With particular interest, it has been found that SLC transporters family is globally expressed in organs and tissues of digestive system. ${ }^{13}$ As transporters facilitative the uptake of small molecules, SLC proteins in digestive system serve as important mediators in maintaining the normal function of absorption of nutrients and ions, ${ }^{14,15}$ excretion of bile acids, ${ }^{16}$ metabolism of toxins, ${ }^{17}$ etc. Given the important role and activity in maintaining the normal daily functions of digestive system, dysfunction of SLC proteins in digestive system may universally mediate the initiation and progression of tumors. In this review, we critically summarized the role and possible mechanism of action of SLC proteins in development of digestive system neoplasms. Differential expression of SLC proteins in tumors of digestive system compared with normal tissue was examined. The possibility of using pharmacologic modulators of SLC proteins in the treatment of digestive system neoplasms was discussed.

\section{Role and regulation of SLC family protein in digestive system neoplasms}

Expressions of SLC family proteins are differential in various types of digestive system neoplasms, and different SLC family proteins may play an opposite role in the same type of digestive system neoplasms. It was noticed in previous studies that SLC family proteins can play either oncogenic or tumor suppressive roles in the carcinogenesis, progression, and metastasis of neoplasms in digestive systems and may mediate cancer cell sensitivity or resistance to chemotherapeutic agents. In addition, more and more studies in retrospective or perspective way revealed the clinical significance of SLC proteins as biomarkers for diagnosis and prognosis of digestive system neoplasms. To further elaborate their functions, we classified SLC families based on their common substrates according to the literature, ${ }^{8}$ with minor modifications.

\section{Metal ion transporters (SLCI I, -39 , and -40 families)}

Holmstrom et al found that SLC11A2 and SLC40A1 were significantly elevated at mRNA levels in hepatocellular carcinoma (HCC) tissues, which may be involved in iron uptake; however, the protein level of SLC40A1 was similar in HCC compared with control liver and did not increase in HCCs exposed to iron. ${ }^{18}$ In colon cancer, expression of iron import machinery protein DMT1, which was encoded by SLC11A2, as well as iron exporter FPN, which was encoded by SLC40A1, was elevated. However, the intracellular location of ferroportin (FPN) suggested reduced iron efflux in colorectal cancers. Iron overload caused by changes in associated gene expression indicated more advanced colon cancer. ${ }^{19}$

The ion transporters have been found to mediate iron metabolism during carcinogenesis. Expression of SLC11A2 and SLC40A1 was proposed to be associated with esophageal carcinogenesis, in which progression of metastatic adenocarcinoma was associated with increased iron deposition. SLC11A2 and SLC40A1 were found to be overexpressed in adenocarcinoma and iron loading to OE33 and SEG-1 cells accelerated cellular proliferation. ${ }^{20}$ In diethylnitrosamineinduced liver adenoma, mice showed dysregulated iron metabolism, which can be associated with altered expression of SLC11A2, suggesting its role in iron metabolism-related carcinogenesis in the liver. ${ }^{21}$ In colon carcinogenesis, it was found that SLC11A2 expression was a critical event in hypoxia-inducible factor $2 \alpha$ (HIF-2 $\alpha$ )-mediated cellular proliferation and cyclin D1 expression, which then initiated neoplastic transformation in colon tissues. ${ }^{22}$ This conclusion was experimentally supported by outcome of another study, in which SLC11A2 was found increased to promoted iron import in lumina. Inhibition of luminal iron can suppress murine intestinal tumorigenesis. ${ }^{23}$ Further study showed that the overexpression of SLC11A2 and downregulation of SLC40A1 in colonic inflammation can exacerbate iron overload which increased risk of colorectal cancer and inflammatory bowel diseases..$^{24}$ Further study suggested that suppression of SLC11A2 may lead to modulation of CDK1-, JAK1-, and STAT3-mediated iron-regulated signaling axis and may slow down the progression of colon carcinogenesis. ${ }^{25}$ 
Ion transporters were also found to mediate oncogenic and tumor-suppressive signaling during cancer progression and metastasis. It was shown that in colorectal cancer cells Caco-2 and SW480, intracellular iron overload caused by increased SLC11A2 and decreased SLC40A1 would promote cell proliferation and metastasis by repressing E-cadherin expression. ${ }^{19} \mathrm{Li}$ et al found that expression of SLC11A2 was associated with hypoxia and reoxygenation in HCC cells. Expression of SLC11A2 increased during hypoxia along with stabilization of HIF- $1 \alpha$, and became not detectable soon after reoxygenation, indicating that HIF- $1 \alpha$ may play a role in mediating SLC11 A2 expression during hypoxia in HCC. ${ }^{26}$ SLC39A6 was identified as an oncogene in HCC. It was found that SLC39A6 was negatively correlated with the outcome of HCC patients. SLC39A6 can promote HCC cell proliferation, migration, and invasion in vitro and induce tumor growth and metastasis in vivo. This may be related to the upregulation of SNAIL and downregulation of E-cadherin in SLC39A6overexpressing $\mathrm{HCC}$ cells. ${ }^{27}$ Consistent results were found in human esophageal carcinoma, in which SLC39A6 expression was higher than non-tumor tissue. Expression of SLC39A6 was correlated with tumor metastasis to lymph node and reduced patient survival time. Knockdown of SLC39A6 reduced tumor cell proliferation in vitro and metastasis in vivo. Overexpression of SLC39A6 elevated intracellular zinc level and activated PI3K/AKT and MAPK1 signaling, leading to invasive phenotype and overexpression of metastasis-related protein such as MMP1, MMP3, Myc, and SLUG. ${ }^{28}$

\section{Transporters of glucose and other sugars (SLC2, -5 , and -45 families)}

In esophageal squamous cell carcinoma, the level of serum atni-SLC2A1 antibodies was significantly elevated in cancer patients compared to healthy donors. Twelve out of 57 (21\%) squamous cell carcinoma patients gained response for antiSLC2A1 antibodies, when a mean $+2 \times$ standard deviation of healthy donors was used as a cut-off level. However, this study did not find out any correlation between anti-SLC2A1 antibody level and clinical pathological factors or survival. ${ }^{29}$ Teng et al found that hepatitis B virus pre-S2 mutant can induce the expression of SLC2A1 via EIF4EBP1, YY1, and MYC dependent manner, which contributed to aberrant glucose uptake and lactate production, thereby promoting tumorigenesis in the liver. ${ }^{30}$ In gastric cancer, SLC2A1 overexpression can promote cellular proliferation and in vitro metastasis and can induce in vivo tumor growth. This was postulated to be associated with SLC2A1-induced glucose utilization of gastric cancer cells. ${ }^{31}$
In gastric cancer, expression of SLC5A8 was epigenetically inactivated, as indicated by the aberrant methylation of SLC5A8 promoter region in 23 of $71(30 \%)$ primary gastric cancer cases. Hypermethylation of SLC5A8 promoter was also found in gastric cell lines, and expression of SLC5A8 can be restored by treatment of 5-aza-2'-deoxycytidine, a methyltransferase inhibitor. Acetylation of histone $\mathrm{H} 3$ in the $5^{\prime}$ region of the gene correlated directly with SLC5A8 expression and inversely with DNA methylation. ${ }^{32}$ Similar observation was found in pancreatic cancer patients. Loss of SLC5A8 was found in all tumor tissue of pancreatic cancer and its promoter region showed dense methylation. Demethylation by aza-deoxycytidine or trichostatin A in pancreatic cancer cells reexpressed SLC5A8. ${ }^{33}$ In a colon cancer model, SLC5A8 was found obligatory for protecting mice from colon cancer induced by low fiber intake. The tumor suppressive role of SLC5A8 was associated with mucosal immune system in response to butyrate, a bacterial metabolite in colon. SLC5A8-null dendritic cells failed to induce Tregs or to suppress interferon- $\gamma$-producing T cells. ${ }^{34}$ SLC5A8 was found to mediate the inhibitory effect of Activin A in colorectal cancer RKO cells. Activin A can induce SLC5A8 expression via transcription activation. Deletion of CAGA elements located within the $-273 /-222$ region of SLC5A8 promoter abolishes its transcription activation by Activin A, and Smad signaling seems to be responsible for Activin A-induced transcription activation of SLC5A8. ${ }^{35}$

Using transcriptome sequencing analysis, Palanisamy et al identified that a gene fusions including SLC45A3 can induce neoplastic transformation of prostate cells. ${ }^{36}$

\section{Transporter of urea, neurotransmitters and biogenic amines, ammonium and choline (SLC22 family)}

Heise et al studied the clinical significance of SLC22A1 and SL22A3 in HCC. It was shown that both SLC22A1 and SLC22A3 were downregulated in HCC samples compared with non-tumor normal liver. Downregulation of SLC22A1 was significantly correlated with more $\mathrm{T} 3$ tumors, larger tumor size, poor differentiation, and high plasma alpha fetoprotein (AFP). Comparatively, HCC with low expression of SLC22A1 gained higher expression of SLC22A3; however, the level of SLC22A3 did not have any clinical correlation. ${ }^{37}$ Another study by Zhu et al also showed that the genetic variant rs7758229 in SLC22A3 has no correlation with the risk of cancer in Chinese population. ${ }^{38}$ Similar observation was obtained by Lauten et al in cholangiocellular carcinoma (CCA), in which SLC22A1 expression was 
negatively correlated with tumor diameter, cancer stages, and patient survival. Expression of SLC22A3 had no significant correlation with tumor characteristics or patient survival in CCA. ${ }^{39}$ Another study was conducted in pancreatic cancer, in which SLC22A1 expression was downregulated while SLC22A3 was upregulated. Expression of SLC22A1 was significantly lower in tumor with angioinvasion, while SLC22A3 expression predicted a longer overall survival in patients with nucleoside analogs treatment. ${ }^{40}$ Jung et al found that expression of SLC22A18 was downregulated in colorectal cancer and inversely correlated with prognosis of patients; ${ }^{41}$ in contrast, SLC22A18 was found to be upregulated in pancreatic cancers. ${ }^{40}$

Some studies have majorly focused on the function of SLC22 family proteins in mediating drug resistance during chemotherapy of cancers. Kitada et al discovered the relationship between SLC22A1 expression and cytotoxicity of platinum treatment to colon cancer cells and found that SLC22A1 may affect the cytotoxicity of oxaliplatin. ${ }^{42}$ In esophageal cancer cells, it was found that expression of SLC22A1 was reduced when cells gained resistance to cisplatin treatment. This was probably mediated by the methylation of SLC22A1 promoter region. Knockdown of SLC22A1 in cisplatinsensitive cells impaired inducible apoptosis. ${ }^{43}$ A subsequent study showed that oxaliplatin toxicity to colon cancer cells may be also related to the expression of SLC22A3. In a retrospective study with 31 patients, Le Roy et al found that SLC22A3 expression was significantly lower in FOLFOX treatment responders, indicating the SLC22A3 remains to be an independent criterion for adjuvant FOLFOX chemotherapy response in colon cancer patients. ${ }^{44}$ High expression of SLC22A3 was correlated with increased lactate dehydrogenase and accumulation of platinum in colon cancer cells after oxaliplatin treatment, indicating increased cytotoxicity. ${ }^{45}$ Qu et al found that SLC22A5 expression was a determinant of oxaliplatin uptake. Expression of SLC22A5 is mediated by peroxisome proliferator-activated receptor gamma (PPAR $\gamma$ ), and upregulation of SLC22A5 by a natural PPAR $\gamma$ agonist luteolin can increase cancer cell sensitivity to oxaliplatin treatment. ${ }^{46}$ Shimakata et al found that patients with AFPproducing gastric cancer had higher expression of SLC22A7, which increased uptake of gemcitabine and fluoropyrimidine, indicating the beneficial role of SLC22A7 to overcome chemoresistance in AFP-producing gastric cancer. ${ }^{47}$ In addition, SLC22 family protein was found to be involved in signaling transduction in cancer cells. Overexpression of SL22A18 induced G2/M cell cycle arrest as well as reduced colony formation of colon cancer cells, and this may inhibit KRAS(G12D)-mediated anchorage-independent growth of cancer cells. Knockdown of Kras promotes SLC22A18, further proving their mutual interaction. ${ }^{41}$

By developing an antibody-drug conjugate ASG-5ME, it was found that pancreatic cancer cell viability, as well as xenografted growth of tumor cells, can be prominently reduced by targeting SLC44A4 in pancreatic cancer cells. ${ }^{48}$

\section{Inorganic cation/anion transporters (SLCI 2, -26, and -34 families)}

In colon cancer, single nucleotide polymorphisms (SNPs) of SLC12A2 were found and showed correlation with a $34 \%$ reduced adenoma risk. ${ }^{49}$ It was found that the Na-K-Cl cotransporter 1 (NKCC1), which was encoded by SLC12A2, is expressed in parenchymal and sinusoidal endothelial cells as well as in hepatic tumor cells. However, expression of SLC12A2 was induced by hyperosmolarity, and induced expression of SLC12A1 in hepatic stellate cells can promote its activation toward myofibroblast-like phenotype. ${ }^{50}$ This was consistent with another study by Zhang et al, in which SLC12A2 was found upregulated in human colon cancer tissue sample using LTQfourier transform mass spectormetry technology (Thermo Fisher Scientific, Waltham, MA, USA). ${ }^{51}$

NKCC1, which was encoded by SLC12A2, was primarily located in the cytoplasm of esophageal carcinoma cells, and depletion of NKCC1 suppressed cell proliferation and rendered G2/M cell cycle arrest. Microarray analysis revealed that knockdown of SLC12A2 initiates G2/M DNA damage pathway-related proteins including MAD2L1, DTL, BLM, CDC20, BRCA1, and E2F5. ${ }^{52} \mathrm{Xu}$ et al suggested that SLC12A5 was significantly overexpressed in colon cancer tissue (78/191, 40.8\%). Overexpression of SLC12A5 can promote cell proliferation, G1/S cell cycle transition and invasion, and suppress the in vitro apoptosis of colon cancer cells. In vivo study showed that SLC12A5 overexpression may promote tumor growth and lung metastasis. The oncogenic activity of SLC12A5 was probably related to the inhibition of apoptosis-inducing factor and endonuclease G-dependent apoptotic signaling pathway, as well as regulation of the key elements of the matrix architecture such as matrix metallopeptidase and fibronectin. This leads to shorter survival in colon cancer patients with high SLC12A5 expression. ${ }^{53}$

Expression of SLC26A3 was also found elevated in the blood sample of colorectal cancer patients, which indicated its potential as a putative biomarker for colon cancer. ${ }^{54,55}$ Pitule et al showed that high expression of SLC26A2 in tumor tissue predicted a longer overall survival and diseasefree interval in colorectal cancer patients, and lower expression of SLC26A2 expression meant longer disease-free interval. ${ }^{56}$ 
Lee et al applied fluorescence in situ hybridization to study the gene fusion of SLC34A2-ROS1 and found patients who had this fusion had poorly differentiated histology with recurrence and died within 2 years of surgery, but this fusion was not an independent prognostic factor for overall survival of gastric cancer patients. ${ }^{57}$ By analyzing gastric cancer samples, Zhang et al found that SLC34A2 expression predicted poor prognosis and tumor recurrence in gastric cancer patients. ${ }^{58}$ SLC34A2 was found overexpressed in HCC cell lines. Knockdown of SLC34A2 can suppress the proliferation, migration, invasion, and epithelial-mesenchymal transition of HCC cells, which may be related to the PI3K/ Akt pathway activity. ${ }^{59}$ Zhang et al reported the involvement of SLC34A2 in the tumor suppressive role of miR-939. SLC34A2 was found to be the direct target of miR-939. The overexpression of SLC34A2 in gastric cancer can activate the oncogenic pathway Ras/Raf/ERK, which may be associated with chemoresistance and recurrence of gastric cancer. ${ }^{58}$

\section{Transporters of nucleotide, amino acid, and oligopeptide (SLC7 and -29 families)}

In the progress of hepatocarcinogenesis induced by chemical carcinogen piperonyl butoxide, expression of SLC7A5 was significantly upregulated in accompany with centrilobular hypertrophy and increased glutathione S-transferase placental form positive foci formation in the liver, as well as reactive oxygen species (ROS) production in liver microsomes. ${ }^{60}$

Some studies found that SLC7 family protein played differential role in mediating cancer progression after tumor was formed. Park et al reported that amino acid transporters SLC38A1 and SLC7A5 might mediate the amino acid metabolism induced by YAP/TAZ in HCC. SLC38A1 and SLC7A5 expressions are inversely correlated with survival of HCC patients, which may be associated with the SLC38A1-/ SLC7A5-induced uptake of amino acid. This increase in amino acid uptake activates $\mathrm{mTORC} 1$ pathway and promotes cell proliferation in $\mathrm{HCC} .{ }^{61}$ Chang et al found that expression of SLC7A11 can be suppressed by 15-keto-PGE2, which in turn reduced intracellular glutathione due to the lack of cysteine and halted cell proliferation and survival of pancreatic cancer cells, suggesting silencing of SLC7A11 as a potential strategy to inhibit pancreatic cells under oxidative stress. ${ }^{62}$

The SLC7 family is also involved in drug resistance of cancer cells. Kishikawa et al discovered the role of SLC7A1 as an arginine transport in mediating resistance of sorafenib in miR-122-silencing HCC cells. SLC7A1 was identified as the direct target of miR-122, the downregulation of which caused overexpression of SLC7A1 and increased level of intracellular arginine. This led to increased nitric oxide level and sorafenib resistance in $\mathrm{HCC}$ cells. ${ }^{63}$

In HCC patients, SLC29A2 expression was significantly associated with advanced stages, vascular invasion, and poor survival. ${ }^{64}$ Expression of SLC29A1 was downregulated in pancreatic cancer and is correlated with tumor angioinvasion. ${ }^{40}$ High expression of SLC29A3 indicated a longer survival of pancreatic cancer patients treated with nucleoside analogs. ${ }^{40}$ Chen et al found that expression of SLC29A2 was upregulated in HCC tissue samples. Knockdown of SLC29A2 can reduce HCC cell proliferation and anchorage-independent growth in vitro, as well as suppress xenografted tumor formation in mice. The mechanism underlying induction of cell proliferation by SLC29A2 overexpression may involve activation of STAT3 signaling pathway. ${ }^{64}$

\section{Organic anion transporters (SLCO family)}

Kinoshita and Miyata found that organic anion transporters C (OATP-C), which is encoded by SLCO1B1, was underexpressed in 55\% (11 cases) of human HCC ${ }^{65}$ However, a controversial observation was made in the study by Cui et al, in which they showed that SLCO1B1 and SLCO1B3 were detectable in primary $\mathrm{HCC}$ but not in CHOL or in liver niches of metastatic colorectal and pancreatic cancers, suggestive of the usefulness of SLCO1B1/SLCO1B3 within a panel of tumor markers for HCC. ${ }^{66}$ This was consistent with the study by Vavricka et al, in which they found that expression of SLCO1B1 was not significantly decreased in HCC, but OATP8 encoded by SLCO1B3 was underexpressed in $60 \%$ of HCC samples compared to adjacent non-tumor liver tissue at both mRNA and protein levels. In addition, the expression level of SLCO1B3 but not SLCO1B1 was inversely correlated with hepatocyte nuclear factor $3 \beta$ (HNF3 $\beta) .{ }^{67}$ Vander Borght et al found that SLCO1B1 and SLCO1B3 were minimally expressed in hepatocellular adenoma, but had high expression In focal nodular hyperplasia of $\mathrm{HCC}$, further indicating its specificity as tumor marker of $\mathrm{HCC} .{ }^{68}$ Vasuri et al found that two organic anion transporters OATP-1B1 and OATP1B3, which were encoded by SLCO1B1 and SLCO1B3, respectively, were inversely correlated with the expression of the biliary type keratins $\mathrm{K} 7$ and $\mathrm{K} 19$, which indicated poor prognosis after liver transplantation in HCC patients. Although they did not observe any correlation between OATP-1B1/1B3 expression and histopathological feature or neoplastic recurrence of HCC after liver transplantation, OATPs, expression 
was found to negatively correlate with HCC-related death after recurrence, suggesting OTAPs as predictive markers in recurrent $\mathrm{HCC} .{ }^{69}$ However, Teft et al found that polymorphism of SLCO1B1 at 388G/G may predict longer progression-free survival (PFS), while SLCO1B3 expression was associated with reduced PFS. ${ }^{70}$ As SLCO1B1-encoded OATP-C was downregulated in HCC, Jung et al studied the mechanism of how bile acid regulates its expression. They found that the transcription of SLCO1B1 was dependent on HNF $1 \alpha$, which was repressed by bile acid supplementation in small heterodimer partner-independent mechanism. ${ }^{71}$ SLCO1B3 but not SLCO1B1 in HCC cells was suppressed by HNF3 $\beta$, which binds to the promoter region at $\mathrm{nt}-39 /-23 .{ }^{67}$

SLCO1B family proteins have been shown to critically participate in cellular uptake of chemotherapeutic agents. Rhodes et al found that gene polymorphisms of SLCO1B1 were involved in irinotecan resistance in metastatic colorectal cancer patients. The combination of at least one SLCO1B1 $521 \mathrm{~T}$ allele, one ABCB1 $1236 \mathrm{C}$ allele, and one UGT1A1*28 variant 7 repeat demonstrated a statistically significant association with grade $3 / 4$ toxicities. ${ }^{72}$ Exploratory analysis showed that polymorphisms of SLCO1B1 can affect the pharmacokinetics and pharmacodynamics of irinotecan, as evidenced by more variability of irinotecan and its active metabolite AUC in patients with SLCO1B1 polymorphisms. ${ }^{73}$ Huang et al found that genotype GA/AA of SNP rs2306283 of SLCO1B1 and genotype GG of SNP rs1051266 of SLC19A1 were associated with a higher rapid response rate of colorectal cancer to irinotecan plus fluoropyrimidine treatment, while SLCO1B1 SNP also predicted longer PFS. ${ }^{74}$ Experimental observation showed that pancreatic cells with high expression of SLCO1B1 and SLCO1B3 were more sensitive to gemcitabine treatment than those with lower expression. ${ }^{75}$

\section{Transporters of vitamins and cofactors (SLC19 family)}

Liu et al found that expression of SLC19A3 was downregulated in various gastric cancer cell lines. Among the panel of seven gastric cancer cell lines, SLC19A3 was suppressed in five (71\%). Hypermethylation of SLC19A3 was found in gastric cancer cell lines $(57 \%, 4 / 7)$, primary gastric carcinoma tissues $(51 \%, 52 / 101)$, and precancerous lesion (intestinal metaplasia) tissues $(32 \%, 8 / 25)$. Demethylation reagent restored expression of SLC19A3. ${ }^{76} \mathrm{Ng}$ et al found that SLC19A3 DNA methylation in plasma was a detectable biomarker in blood samples of gastric cancer patients, and its level was effective in differentiating gastric cancer from healthy subjects. In addition, this potential biomarker was useful in predicting both early and late-stage tumors. ${ }^{77}$ Ikehata et al analyzed 12 SLC transporters possessing cytosinephosphate-guanine islands and found that SLC19A3 showed correlation between its mRNA level and DNA methylation status. Demethylation treatment increased histone H3 acetylation of SLC19A3 promoter region in colorectal cancer cell lines and initiated SLC19A3 expression. ${ }^{78}$ Reexpression of SLC19A3 inhibited growth of gastric cancer cells. ${ }^{76}$ SNP was also found in SLC19A1, the solute carrier in the same family; however, its correlation with risk of colorectal cancer was not obvious. ${ }^{79}$

\section{Mitochondrial carrier (SLC25 family)}

Chen et al applied gel-assisted digestion and iTRAQ labeling MS to identifying membrane proteins with variable expression in colorectal cancer patients. Mitochondrial SLC25A was found to be one of the potential biomarkers that are overexpressed in cancer. ${ }^{80}$ Wong et al discovered the function and regulation of SLC25A22 in human colorectal cancer. SLC25A22 expression was inversely associated with shorter survival of patients. Knockdown of SLC25A22 in KRAS-mutant colon cancer cells suppressed proliferation, migration, and invasion in vitro and reduced formation and metastasis of tumor in vivo. Mechanically, SLC25A22 promotes biosynthesis of aspartate, which promoted cell proliferation and reduced apoptosis via GOT1. The increased oxaloacetate level by SLC25A22/GOT1 led to regeneration of oxidized nicotinamide adenine dinucleotide and nicotinamide adenine dinucleotide phosphate, which promoted glycolysis and produced adenosine triphosphate. This caused activation of ERK pathway due to the reduced activity of AMPK. ${ }^{81}$

\section{Expression of SLC family proteins in digestive system neoplasms}

To further understand the clinically relevant roles of SLC family proteins in development of digestive system neoplasms, we extracted protein expression data from human cancer database GEPIA. ${ }^{82}$ Data of expression of proteins from the aforementioned SLC families in CHOL, colon adenocarcinoma, esophageal carcinoma, liver hepatocellular carcinoma, pancreatic adenocarcinoma, rectum adenocarcinoma, and stomach adenocarcinoma were collected and analyzed. Data were present in the form of logarithmic values of fold change of protein expression in tumors to that in normal tissue. Heatmap was generated (Figure 1), and it was found that SLC family proteins do not always exhibit consistent profile of expression within different tumors of digestive systems, for instance, expression of transporters 
A

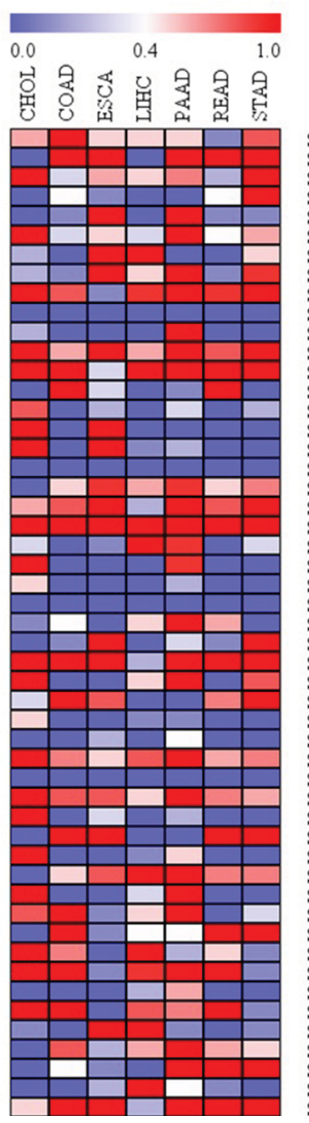

B

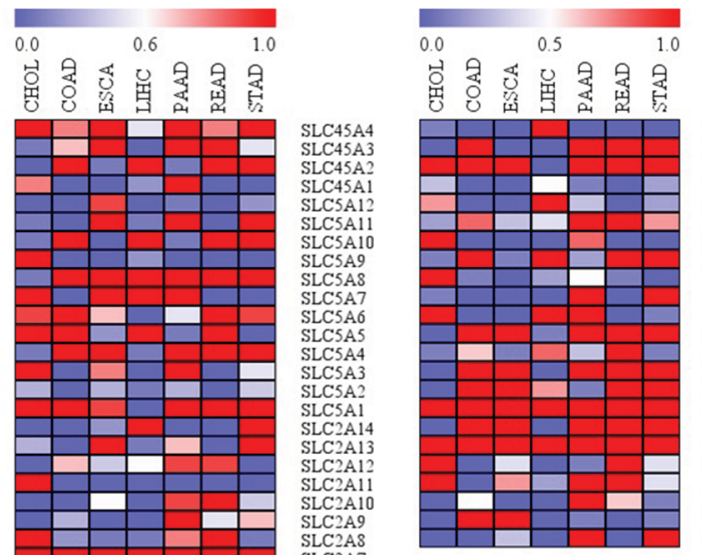

$\mathbf{F}$

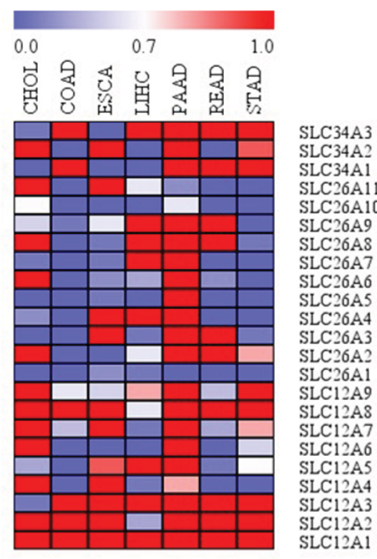

G

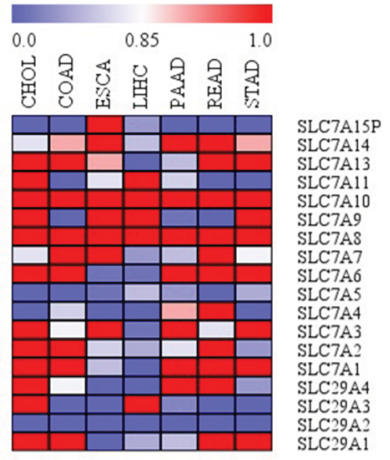

Figure I Differential expressions of SLC family proteins in digestive system neoplasms.

Notes: (A) Session patterns of SLC25 and SLC19 family in digestive neoplasm samples; (B) Expression patterns of SLC45, SLC5 and SLC2 family in digestive neoplasm samples; (C) Expression patterns of SLCO family in digestive neoplasm samples; (D) Expression patterns of SLC22 family in digestive neoplasm samples; (E) Expression patterns of SLC39 and SLCI I family in digestive neoplasm samples; (F) Expression patterns of SLC26 and SLCI2 family in digestive neoplasm samples; (G) Expression patterns of SLC7 and SLC29 family in digestive neoplasm samples.

Abbreviations: SLC, solute carrier; CHOL, cholangiocarcinoma; COAD, colon adenocarcinoma; ESCA, esophageal carcinoma; LIHC, liver hepatocellular carcinoma; PAAD, pancreatic adenocarcinoma; READ, rectum adenocarcinoma; STAD, stomach adenocarcinoma.

of urea, neurotransmitters and biogenic amines, ammonium and choline (Figure 1D), while expression of metal ion transporters varies in different tumors and has higher consistency in their expression profiles in different tumors (Figure 1E). Meanwhile, proteins in the same SLC family did not have similar trend of either overexpression or downregulation in digestive system tumors. Here we have discussed some particular SLC proteins with significant fold changes in tumor.

\section{SLC family proteins with consistent expression profile in different tumors of digestive system}

It was particularly noticed that some family proteins are globally overexpressed or downregulated across different types of digestive system neoplasms, indicating that the oncogenic or tumor suppressive roles of these proteins are not tissue specific and may be associated with their physiological functions at cellular level. For instance, most of the proteins in the SLC39 family are overexpressed in tumors of digestive systems. SLC39 family is composed of zinc transporters, which are also recognized as ZRT, IRT-like proteins. SLC39 proteins primarily mediate the influx of zinc into cytoplasm, from either extracellular region or subcellular organelles, and are the important factors in maintaining the zinc homeostasis of the cells. ${ }^{83}$ Although different SLC39 proteins vary in their abundance in different tissues, they globally increase the cellular uptake of zinc, which could be energy-dependent or -independent. ${ }^{84}$ Although a previous study has shown that uptake of zinc may be good for cancer prevention, there are a lot of clues suggestive of the protumoral effect of high levels of cytosolic zinc. ${ }^{85}$ In gastrointestinal cancers, gastrins are particularly identified as oncogenic factor that accelerates cancer development. It was found that the promoter region of gastrins was zinc-responsive, and high level of intracellular zinc could initiate transcription of gastrins by stimulating Erk1/2 pathway, which suggested the oncogenic role of intracellular zinc in neoplasms of digestive systems. ${ }^{86}$ High extracellular zinc environment caused uptake of zinc, which promoted the aggressiveness of cancer cells by increasing its proliferation and invasion. ${ }^{87}$ In cancer cells with acquired 
tamoxifen resistance, the intracellular zinc level was markedly increased, which can be associated with induced expression of SLC39A7. The high intracellular zinc level then promoted the growth of cancer cells by initiating EGFR signaling. ${ }^{88}$ Activation of zinc-sensing receptors may contribute to the chemoresistance of cancer cells by activating GPR $39 .{ }^{89}$ Low zinc environment was found to induce intracellular stress, and chronic zinc deficiency can cause changes in mitochondrial activity without significantly altering DNA damage and cell death, which thus induced cell senescence and growth inhibition..$^{90}$ Furthermore, zinc deficiency in cancer cells may lead to a shift toward phenotype of less malignancy, with presentation of enhanced apoptosis and reduced proliferation and invasion. ${ }^{91}$ Depletion of intracellular zinc can reduce the migratory and invasive ability of cancer cells. ${ }^{92}$ This can be mediated by SLC39A6 and SLC39A10, ${ }^{93}$ both of which are globally overexpressed in neoplasms of digestive system (Figure 1E). Zinc deficiency in cellular level can promote cancer cell apoptosis, while reexpression of SLC39A4, which restored intracellular zinc, conferred resistance of cancer to cell cycle arrest and apoptosis. ${ }^{94}$ Miyai et al also observed that SLC39A10 exerted similar function to reduce cell apoptosis. ${ }^{95}$ These findings suggest that maintenance of high intracellular level of zinc, which is possibly associated with overexpression of SLC39 family in neoplasms of digestive system, may have global effect on tumor growth and progression regardless of the cancer type. Other SLC family proteins that exhibit consistent expression profile in different neoplasms of digestive systems may be discussed in other future reviews.

\section{SLC family proteins with unique expression profile in particular tumors of digestive system}

A few of SLC family proteins exhibit unique expression profile in particular tissues, such as liver, bile duct, and esophagus. This can be either a particular change in the expression of SLC proteins in specific tissues or proteins have special expression pattern in particular tissues when compared with its patterns in other tissue. This could be suggestive of association of change in the expression of particular SLC protein with the specific function of that tissue. For instance, it was found that several members of SLC22 family such as SLC22A10, SLC22A9, and SLC22A7 are downregulated in CHOL, a cancer of bile duct that is composed of mutated epithelial cells. ${ }^{96}$ SLC22 family encodes OATs proteins that transport hydrophilic organic anions.$^{97}$ A previous study has showed that normative expression of SLC22 family protein is important for the formation and secretion of bile acid from liver to small intestine. ${ }^{98}$ SLC22 expression in basolateral membrane contributed to the bile salt transport. ${ }^{99}$ Although there is still no direct scientific evidence to show that knockdown of SLC22 can cause CHOL, it is postulated that abnormal bile acid secretion and formation caused by SLC22 loss may be associated with bile duct carcinogenesis by mutated cells.

\section{Pharmacologic modulation of SLC protein family in the treatment of tumors of digestive system}

Regarding the critical role of SLC proteins in mediating the initiation, progression, and metastasis of different cancers, which has been critically reviewed elsewhere ${ }^{100}$ and in our present review, pharmacologic modulations on the expressions and activities of SLC proteins might deliver potential therapeutic activities on cancers. A series of small molecule modulators of SLC proteins have been discovered with high-throughput screening of large compound libraries, most of which were found to be inhibitors of transporters function. ${ }^{101,102}$ Lin et al reviewed the small molecules that can modulate SLC proteins, in the extent of both activation and inhibition, ${ }^{103}$ some of which have been reported to suppress tumors of digestive system. Sulfasalazine, a SLC7A11 inhibitor originally developed for arthritis, exhibited preventive effect in the carcinogenesis of colon cancer. ${ }^{104,105}$ It was also reported to sensitize cells of pancreatic and colorectal cancers in response to chemotherapeutic treatments, such as cisplatin, ${ }^{106} 5$-fluouracil,,${ }^{107}$ and gemcitabine. ${ }^{108}$ SLC18A2 inhibitors such as amphetamine and reserpine were found to prevent hepatocarcinogenesis in experimental animals. ${ }^{109-111}$ This can be related to their modulation on Bcl-2 family and p53. ${ }^{12}$ Clodronate, a common inhibitor of SLC25A4, SLC25A5, and SLC25A6, was encapsulated into liposome to be specifically delivered to macrophage. Liposome clodronate can remove systemic macrophages. ${ }^{113}$ Treatment of liposome-encapsulated clodronate can suppress the growth and metastasis of pancreatic cancer and colorectal cancer in experimental animal models ${ }^{14,115}$ and enhance the therapeutic outcome of sorafenib in HCC. ${ }^{116}$ SLC6A2 inhibitors, including sertraline and fluoxetine, exhibited prominent antitumor effects. Gil-Ad et al found that sertraline can suppress the growth of colon tumor and induce cancer cell apoptosis without significant toxicity. ${ }^{17}$ Fluoxetine suppressed nuclear factor- $\mathrm{KB}$-associated inflammation in colitis and reduced risk of colon cancer incidence, ${ }^{117}$ and this oncostatic effect of fluoxetine can be probably due to its modulatory activity on 5-hydroxytryptamine metabolism. ${ }^{118}$ 
Fluoxetine was also found to suppress the proliferation of colon cancer cells and induce cell cycle arrest. ${ }^{119,120}$ It can also induce apoptosis of HCC cells via initiating ROS-induced caspase-3 activation. ${ }^{121,122}$ Inhibitors of SLC12A1, such as bumetanide and furosemide, were found to diminish tumor growth by inducing cell cycle arrest and inhibiting tumor angiogenesis. ${ }^{123,124}$ SLC1A3 activator riluzole can suppress the proliferation of HCC cells by elevating intracellular ROS production. ${ }^{125}$ These findings suggest that particular modulators (Figure 2) of SLC family proteins may be potential nextgeneration therapeutic agents for digestive system neoplasms.

\section{Significance of studying SLC proteins in digestive system neoplasms}

Our study comes out with several significances. First, the idea of studying different SLC proteins initiated from the observation that expression of different SLC proteins has different patterns in digestive system neoplasms. Presence of SLC proteins in normal tissue and organs of digestive system has its physiological function in maintaining the daily operation of the system, as digestive tract is the largest internal system communicating and interacting with foreign materials such as food, chemicals, and toxins, which are generally mediated by SLC proteins. Mutation or dysfunction of SLC proteins is, therefore, a risk factor for the occurrence of gastrointestinal malignancies, or it mediates the progression of cancers.
In this study, we summarized the different roles of SLC proteins in gastrointestinal malignancies, which highlighted the knowledge in understanding SLC proteins-associated disease development, so that the diagnostic and therapeutic value of SLC protein target therapy can be further discussed. Second, an in-depth analysis has been conducted in this study by not only summarizing the narrative texts describing the role of SLC protein in the development of digestive cancers but also carrying out quantitative analysis on the expression patterns of SLC proteins in different digestive cancers. The analysis on the expression patterns of SLC proteins predicts their particular functions by reviewing related mechanistic studies on the substrates that SLC proteins transport. And a graphic summary on the cellular signaling of cancer cells being regulated by SLC proteins elaborated the underlying interactions between cellular molecules and SLC substrates. These findings indicate that SLC superfamily would likely be a suitable target in pathogenesis identification, target therapy discovery, and therapeutic outcome prediction. Third, the illustration of SLC proteins in mediating drug resistance of chemotherapy in digestive system neoplasms would also lead to a great significance in medical extent, as profiling on the expression of drug resistance-related SLC genes may result in a better understanding of therapeutic outcome of a particular cancer patient. In this study, we figured out the actions of different SLC proteins as chemotherapeutics transporters in tumor cells of digestive neoplasms. This global picture

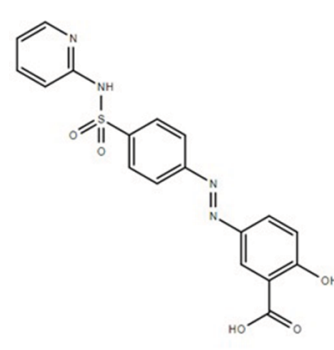

Sulfasalazine

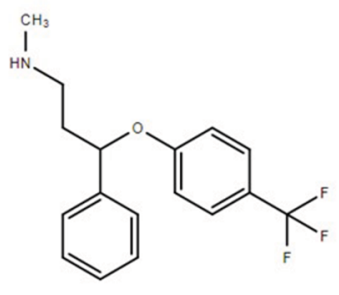

Fluoxetine

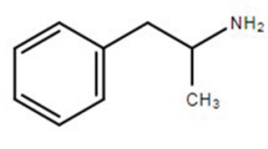

Amphetamine

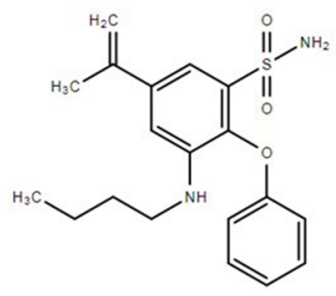

Bumetanide

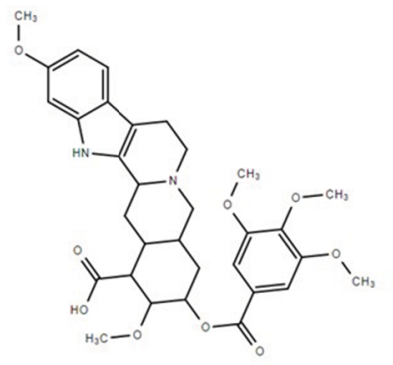

Reserpine

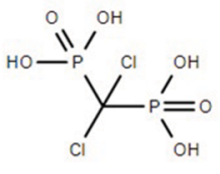

Clodronate

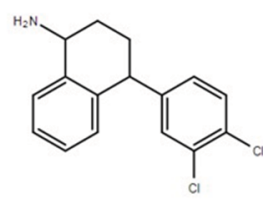

Sertraline

Figure 2 Small molecule modulators of SLC proteins with anticancer potential. Abbreviation: SLC, solute carrier. 


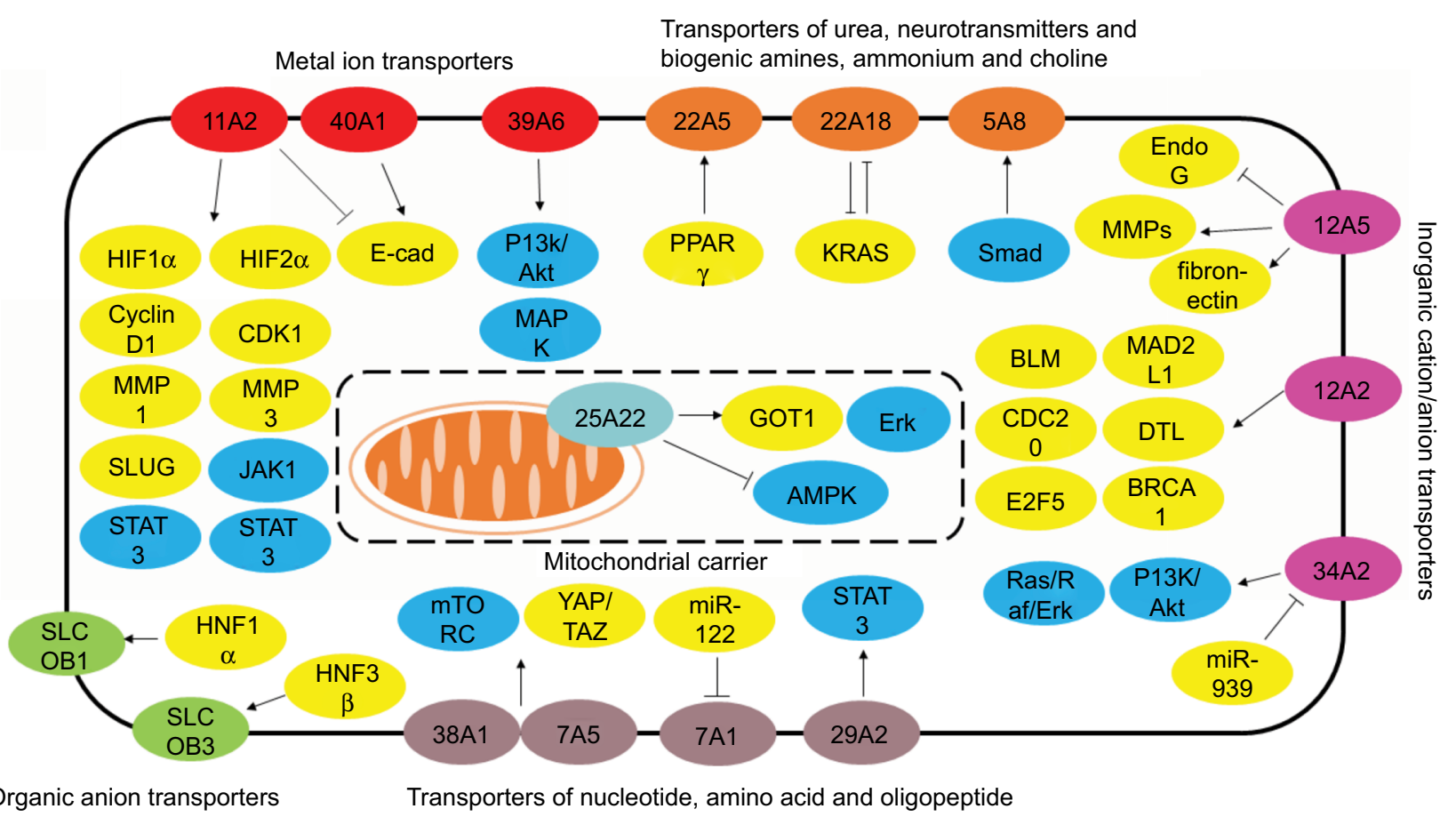

Organic anion transporters

Transporters of nucleotide, amino acid and oligopeptide

Figure 3 Overall scheme of the regulation of SLC protein expression and signaling transduction.

Abbreviation: SLC, solute carrier.

in combination with pharmacogenomic approaches on the actual expression of SLC proteins in tumor tissues of cancer patients will be helpful information for predicting the therapeutic outcome of treatment as well as designing reasonable and personalized treatment. And last but not least, in this study, we also summarized the possible signaling transduction and oncomolecules that interact with SLC proteins. Although the function of SLC proteins is to transport ions/ chemicals through cell membrane, which definitely results in signal transduction at cellular level, the illustration of global picture of SLC protein-regulated signaling pathway and oncomolecules will be helpful in identifying the key mechanism involved in the tumor promoting/reducing effect of SLC modulators. This will not only facilitate the discovery of high-efficacy SLC modulators for cancer treatment but also potentiate the possibility of development of novel therapeutic regimens of SLC modulators with other target therapeutics for a better efficacy of cancer treatment.

\section{Conclusion}

SLC family proteins are a group of transporters of small molecule substrates that play important physiological role in maintaining the normal function of human body, including digestive system. Changes in expression and regulation of SLC family proteins (as summarized in Figure 3) were observed in neoplasms of digestive system and their dysregulation may be associated with increased carcinogenesis, tumor progression, and metastasis, as well as with resistance of cancer cells to chemotherapeutic agents. The role of SLC family proteins in neoplasms of digestive system can be dependent on their dysfunction in transporting substrates or be target specific. Several pharmacologic modulators of SLC family proteins were found to deliver potent antitumor activity on neoplasms of digestive system. This review highlighted the potential of SLC family proteins as drug target for the treatment of digestive system neoplasms.

\section{Acknowledgment}

This work was financially supported by the grant from National Nature Science Foundation of China (NSFC, Grant No. 81403248).

\section{Disclosure}

The authors report no conflicts of interest in this work.

\section{References}

1. Torre LA, Bray F, Siegel RL, Ferlay J, Lortet-Tieulent J, Jemal A. Global cancer statistics, 2012. CA Cancer J Clin. 2015;65(2):87-108.

2. Li ZS, Li Q. [The latest $2010 \mathrm{WHO}$ classification of tumors of digestive system]. Zhonghua Bing Li Xue Za Zhi. 2011;40(5):351-354. Chinese.

3. Fu M, Zou C, Pan L, et al. Long noncoding RNAs in digestive system cancers: functional roles, molecular mechanisms, and clinical implications (review). Oncol Rep. 2016;36(3):1207-1218. 
4. Marusyk A, Polyak K. Tumor heterogeneity: causes and consequences. Biochim Biophys Acta. 2010;1805(1):105-117.

5. Yang NJ, Hinner MJ. Getting across the cell membrane: an overview for small molecules, peptides, and proteins. Methods Mol Biol. 2015;1266:29-53.

6. International Transporter Consortium, Giacomini KM, Huang SM, et al. Membrane transporters in drug development. Nat Rev Drug Discov. 2010;9(3):215-236.

7. Singh H, Velamakanni S, Deery MJ, Howard J, Wei SL, van Veen HW. ATP-dependent substrate transport by the ABC transporter MsbA is proton-coupled. Nat Commun. 2016;7:12387.

8. He L, Vasiliou K, Nebert DW. Analysis and update of the human solute carrier (SLC) gene superfamily. Hum Genom. 2009;3(2):195-206.

9. Li Q, Shu Y. Role of solute carriers in response to anticancer drugs. Mol Cell Ther. 2014;2:15.

10. Diallinas G. Understanding transporter specificity and the discrete appearance of channel-like gating domains in transporters. Front Pharmacol. 2014;5:207.

11. Colas C, Ung PM, Schlessinger A. SLC transporters: structure, function, and drug discovery. Medchemcomm. 2016;7(6):1069-1081.

12. Nigam SK. What do drug transporters really do? Nat Rev Drug Discov. 2015;14(1):29-44.

13. Li Y, Lu J, Paxton JW. The role of ABC and SLC transporters in the pharmacokinetics of dietary and herbal phytochemicals and their interactions with xenobiotics. Curr Drug Metab. 2012;13(5):624-639.

14. Gulec S, Anderson GJ, Collins JF. Mechanistic and regulatory aspects of intestinal iron absorption. Am J Physiol Gastrointest Liver Physiol. 2014;307(4):G397-G409.

15. Chan K, Busque SM, Sailer M, et al. Loss of function mutation of the Slc38a3 glutamine transporter reveals its critical role for amino acid metabolism in the liver, brain, and kidney. Pflugers Arch. 2016;468(2):213-227.

16. Suga T, Yamaguchi H, Sato T, Maekawa M, Goto J, Mano N. Preference of conjugated bile acids over unconjugated bile acids as substrates for OATP1B1 and OATP1B3. PLoS One. 2017;12(1):e0169719.

17. Sato T, Yamaguchi H, Kogawa T, Abe T, Mano N. Organic anion transporting polypeptides $1 \mathrm{~B} 1$ and $1 \mathrm{~B} 3$ play an important role in uremic toxin handling and drug-uremic toxin interactions in the liver. J Pharm Pharm Sci. 2014;17(4):475-484.

18. Holmstrom P, Gafvels M, Eriksson LC, et al. Expression of iron regulatory genes in a rat model of hepatocellular carcinoma. Liver Int. 2006;26(8):976-985.

19. Brookes MJ, Hughes S, Turner FE, et al. Modulation of iron transport proteins in human colorectal carcinogenesis. Gut. 2006;55(10):1449-1460.

20. Boult J, Roberts K, Brookes MJ, et al. Overexpression of cellular iron import proteins is associated with malignant progression of esophageal adenocarcinoma. Clin Cancer Res. 2008;14(2):379-387.

21. Youn P, Kim S, Ahn JH, Kim Y, Park JD, Ryu DY. Regulation of iron metabolism-related genes in diethylnitrosamine-induced mouse liver tumors. Toxicol Lett.2009;184(3):151-158.

22. Xue X, Taylor M, Anderson E, et al. Hypoxia-inducible factor-2alpha activation promotes colorectal cancer progression by dysregulating iron homeostasis. Cancer Res. 2012;72(9):2285-2293

23. Radulescu S, Brookes MJ, Salgueiro P, et al. Luminal iron levels govern intestinal tumorigenesis after Apc loss in vivo. Cell Rep. 2012;2(2):270-282.

24. Chua AC, Klopcic BR, Ho DS, et al. Dietary iron enhances colonic inflammation and IL-6/IL-11-Stat3 signaling promoting colonic tumor development in mice. PLoS One. 2013;8(11):e78850.

25. Xue X, Ramakrishnan SK, Weisz K, et al. Iron uptake via DMT1 integrates cell cycle with JAK-STAT3 signaling to promote colorectal tumorigenesis. Cell Metab. 2016;24(3):447-461.

26. Li Z, Lai Z, Ya K, et al. Correlation between the expression of divalent metal transporter 1 and the content of hypoxia-inducible factor-1 in hypoxic HepG2 cells. J Cell Mol Med. 2008;12(2):569-579.
27. Lian J, Jing Y, Dong Q, et al. miR-192, a prognostic indicator, targets the SLC39A6/SNAIL pathway to reduce tumor metastasis in human hepatocellular carcinoma. Oncotarget. 2016;7(3):2672-2683.

28. Cheng X, Wei L, Huang X, et al. Solute carrier family 39 member 6 gene promotes aggressiveness of esophageal carcinoma cells by increasing intracellular levels of zinc, activating phosphatidylinositol 3-kinase signaling, and up-regulating genes that regulate metastasis. Gastroenterology. 2017;152(8):1985-1997.e12.

29. Kuboshima M, Shimada H, Liu TL, et al. Identification of a novel SEREX antigen, SLC2A1/GLUT1, in esophageal squamous cell carcinoma. Int J Oncol. 2006;28(2):463-468.

30. Teng CF, Hsieh WC, Wu HC, et al. Hepatitis B virus pre-S2 mutant induces aerobic glycolysis through mammalian target of rapamycin signal cascade. PLoS One. 2015;10(4):e0122373.

31. Yan S, Wang Y, Chen M, Li G, Fan J. Deregulated SLC2A1 promotes tumor cell proliferation and metastasis in gastric cancer. Int J Mol Sci. 2015;16(7):16144-16157.

32. Ueno M, Toyota M, Akino K, et al. Aberrant methylation and histone deacetylation associated with silencing of SLC5A8 in gastric cancer Tumour Biol. 2004;25(3):134-140.

33. Park JY, Helm JF, Zheng W, et al. Silencing of the candidate tumor suppressor gene solute carrier family 5 member 8 (SLC5A8) in human pancreatic cancer. Pancreas. 2008;36(4):e32-e39.

34. Gurav A, Sivaprakasam S, Bhutia YD, Boettger T, Singh N, Ganapathy V. Slc5a8, a Na+-coupled high-affinity transporter for short-chain fatty acids, is a conditional tumour suppressor in colon that protects against colitis and colon cancer under low-fibre dietary conditions. Biochem J. 2015;469(2):267-278.

35. Zhang Y, Bao YL, Yang MT, et al. Activin A induces SLC5A8 expression through the Smad3 signaling pathway in human colon cancer RKO cells. Int J Biochem Cell Biol. 2010;42(12):1964-1972.

36. Palanisamy N, Ateeq B, Kalyana-Sundaram S, et al. Rearrangements of the RAF kinase pathway in prostate cancer, gastric cancer and melanoma. Nat Med. 2010;16(7):793-798.

37. Heise M, Lautem A, Knapstein J, et al. Downregulation of organic cation transporters OCT1 (SLC22A1) and OCT3 (SLC22A3) in human hepatocellular carcinoma and their prognostic significance. BMC Cancer. 2012;12:109.

38. Zhu L, Du M, Gu D, et al. Genetic variant rs 7758229 in 6q26-q27 is not associated with colorectal cancer risk in a Chinese population. PLoS One. 2013;8(3):e59256.

39. Lautem A, Heise M, Grasel A, et al. Downregulation of organic cation transporter 1 (SLC22A1) is associated with tumor progression and reduced patient survival in human cholangiocellular carcinoma. Int $J$ Oncol. 2013;42(4):1297-1304.

40. Mohelnikova-Duchonova B, Brynychova V, Hlavac V, et al. The association between the expression of solute carrier transporters and the prognosis of pancreatic cancer. Cancer Chemother Pharmacol. 2013;72(3):669-682.

41. Jung Y, Jun Y, Lee HY, et al. Characterization of SLC22A18 as a tumor suppressor and novel biomarker in colorectal cancer. Oncotarget. 2015;6(28):25368-25380.

42. Kitada N, Takara K, Minegaki T, et al. Factors affecting sensitivity to antitumor platinum derivatives of human colorectal tumor cell lines. Cancer Chemother Pharmacol. 2008;62(4):577-584.

43. Lin R, Li X, Li J, et al. Long-term cisplatin exposure promotes methylation of the OCT1 gene in human esophageal cancer cells. Dig Dis Sci. 2013;58(3):694-698.

44. Le Roy B, Tixier L, Pereira B, et al. Assessment of the relation between the expression of oxaliplatin transporters in colorectal cancer and response to FOLFOX-4 adjuvant chemotherapy: a case control study. PLoS One. 2016;11(2): 0148739.

45. Yokoo S, Masuda S, Yonezawa A, Terada T, Katsura T, Inui K. Significance of organic cation transporter 3 (SLC22A3) expression for the cytotoxic effect of oxaliplatin in colorectal cancer. Drug Metab Dispos. 2008;36(11):2299-2306. 
46. Qu Q, Qu J, Guo Y, Zhou BT, Zhou HH. Luteolin potentiates the sensitivity of colorectal cancer cell lines to oxaliplatin through the PPARgamma/OCTN2 pathway. Anticancer Drugs. 2014;25(9): 1016-1027.

47. Shimakata T, Kamoshida S, Kawamura J, et al. Immunohistochemical expression profiles of solute carrier transporters in alphafetoprotein-producing gastric cancer. Histopathology. 2016;69(5): 812-821.

48. Mattie M, Raitano A, Morrison K, et al. The discovery and preclinical development of ASG-5ME, an antibody-drug conjugate targeting SLC44A4-positive epithelial tumors including pancreatic and prostate cancer. Mol Cancer Ther. 2016;15(11):2679-2687.

49. Zhu X, Liang J, Shrubsole MJ, et al. Calcium intake and ion transporter genetic polymorphisms interact in human colorectal neoplasia risk in a 2-phase study. $J$ Nutr. 2014;144(11):1734-1741.

50. Schliess F, Schafer C, vom Dahl S, Fischer R, Lordnejad MR, Haussinger D. Expression and regulation of the $\mathrm{Na}(+) / \mathrm{K}(+) / 2 \mathrm{Cl}(-)$ cotransporter NKCC1 in rat liver and human HuH-7 hepatoma cells. ArchBiochem Biophys. 2002;401(2):187-197.

51. Zhang Y, Liu Y, Ye Y, et al. Quantitative proteome analysis of colorectal cancer-related differential proteins. J Cancer Res Clin Oncol. 2017;143(2):233-241.

52. Shiozaki A, Nako Y, Ichikawa $\mathrm{D}$, et al. Role of the $\mathrm{Na}(+) / \mathrm{K}(+) / 2 \mathrm{Cl}(-)$ cotransporter NKCC1 in cell cycle progression in human esophageal squamous cell carcinoma. World J Gastroenterol. 2014;20(22): 6844-6859.

53. Xu L, Li X, Cai M, et al. Increased expression of solute carrier family 12 member 5 via gene amplification contributes to tumour progression and metastasis and associates with poor survival in colorectal cancer. Gut. 2016;65(4):635-646.

54. Lauriola M, Ugolini G, Rosati G, et al. Identification by a Digital Gene Expression Displayer (DGED) and test by RT-PCR analysis of new mRNA candidate markers for colorectal cancer in peripheral blood. Int J Oncol. 2010;37(2):519-525.

55. Ostasiewicz B, Ostasiewicz P, Dus-Szachniewicz K, Ostasiewicz $\mathrm{K}$, Ziolkowski P. Quantitative analysis of gene expression in fixed colorectal carcinoma samples as a method for biomarker validation. Mol Med Rep. 2016;13(6):5084-5092.

56. Pitule P, Vycital O, Bruha J, et al. Differential expression and prognostic role of selected genes in colorectal cancer patients. Anticancer Res. 2013;33(11):4855-4865

57. Lee J, Lee SE, Kang SY, et al. Identification of ROS1 rearrangement in gastric adenocarcinoma. Cancer. 2013;119(9):1627-1635.

58. Zhang JX, Xu Y, Gao Y, et al. Decreased expression of miR-939 contributes to chemoresistance and metastasis of gastric cancer via dysregulation of SLC34A2 and Raf/MEK/ERK pathway. Mol Cancer. 2017;16(1):18.

59. Li Y, Chen X, Lu H. Knockdown of SLC34A2 inhibits hepatocellular carcinoma cell proliferation and invasion. Oncol Res. 2016;24(6): 511-519.

60. Muguruma M, Unami A, Kanki M, et al. Possible involvement of oxidative stress in piperonyl butoxide induced hepatocarcinogenesis in rats. Toxicology. 2007;236(1-2):61-75.

61. Park YY, Sohn BH, Johnson RL, et al. Yes-associated protein 1 and transcriptional coactivator with PDZ-binding motif activate the mammalian target of rapamycin complex 1 pathway by regulating amino acid transporters in hepatocellular carcinoma. Hepatology. 2016;63(1):159-172.

62. Chang EY, Chang YC, Shun CT, et al. Inhibition of prostaglandin reductase 2, a putative oncogene overexpressed in human pancreatic adenocarcinoma, induces oxidative stress-mediated cell death involving $\mathrm{xCT}$ and $\mathrm{CTH}$ gene expressions through 15-keto-PGE2. PLoS One. 2016;11(1):e0147390.

63. Kishikawa T, Otsuka M, Tan PS, et al. Decreased miR122 in hepatocellular carcinoma leads to chemoresistance with increased arginine. Oncotarget. 2015;6(10):8339-8352.
64. Chen CF, Hsu EC, Lin KT, et al. Overlapping high-resolution copy number alterations in cancer genomes identified putative cancer genes in hepatocellular carcinoma. Hepatology. 2010;52(5):1690-1701.

65. Kinoshita M, Miyata M. Underexpression of mRNA in human hepatocellular carcinoma focusing on eight loci. Hepatology. 2002;36(2):433-438.

66. Cui Y, Konig J, Nies AT, et al. Detection of the human organic anion transporters SLC21A6 (OATP2) and SLC21A8 (OATP8) in liver and hepatocellular carcinoma. Lab Invest. 2003;83(4):527-538.

67. Vavricka SR, Jung D, Fried M, Grutzner U, Meier PJ, Kullak-Ublick GA. The human organic anion transporting polypeptide 8 (SLCO1B3) gene is transcriptionally repressed by hepatocyte nuclear factor 3 beta in hepatocellular carcinoma. J Hepatol. 2004;40(2):212-218.

68. Vander Borght S, Libbrecht L, Blokzij1 H, et al. Diagnostic and pathogenetic implications of the expression of hepatic transporters in focal lesions occurring in normal liver. J Pathol. 2005;207(4):471-482.

69. Vasuri F, Golfieri R, Fiorentino M, et al. OATP 1B1/1B3 expression in hepatocellular carcinomas treated with orthotopic liver transplantation. Virchows Arch. 2011;459(2):141-146.

70. Teft WA, Welch S, Lenehan J, et al. OATP1B1 and tumour OATP1B3 modulate exposure, toxicity, and survival after irinotecan-based chemotherapy. Br J Cancer. 2015;112(5):857-865.

71. Jung D, Kullak-Ublick GA. Hepatocyte nuclear factor 1 alpha: a key mediator of the effect of bile acids on gene expression. Hepatology. 2003;37(3):622-631.

72. Rhodes KE, Zhang W, Yang D, et al. ABCB1, SLCO1B1 and UGT1A1 gene polymorphisms are associated with toxicity in metastatic colorectal cancer patients treated with first-line irinotecan. Drug Metab Lett. 2007;1(1):23-30.

73. Innocenti F, Kroetz DL, Schuetz E, et al. Comprehensive pharmacogenetic analysis of irinotecan neutropenia and pharmacokinetics. J Clin Oncol. 2009;27(16):2604-2614.

74. Huang L, Zhang T, Xie C, et al. SLCO1B1 and SLC19A1 gene variants and irinotecan-induced rapid response and survival: a prospective multicenter pharmacogenetics study of metastatic colorectal cancer. PLoS One. 2013;8(10):e 77223.

75. Kounnis V, Chondrogiannis G, Mantzaris MD, et al. Microcystin LR shows cytotoxic activity against pancreatic cancer cells expressing the membrane OATP1B1 and OATP1B3 transporters. Anticancer Res. 2015;35(11):5857-5865.

76. Liu X, Lam EK, Wang X, et al. Promoter hypermethylation mediates downregulation of thiamine receptor SLC19A3 in gastric cancer. Tumour Biol. 2009;30(5-6):242-248.

77. Ng EK, Leung CP, Shin VY, et al. Quantitative analysis and diagnostic significance of methylated SLC19A3 DNA in the plasma of breast and gastric cancer patients. PLoS One. 2011;6(7):e22233.

78. Ikehata M, Ueda K, Iwakawa S. Different involvement of DNA methylation and histone deacetylation in the expression of solute-carrier transporters in 4 colon cancer cell lines. Biol Pharm Bull. 2012;35(3):301-307.

79. Figueiredo JC, Levine AJ, Lee WH, et al. Genes involved with folate uptake and distribution and their association with colorectal cancer risk. Cancer Causes Control. 2010;21(4):597-608.

80. Chen JS, Chen KT, Fan CW, et al. Comparison of membrane fraction proteomic profiles of normal and cancerous human colorectal tissues with gel-assisted digestion and iTRAQ labeling mass spectrometry. FEBS J. 2010;277(14):3028-3038.

81. Wong CC, Qian Y, Li X, et al. SLC25A22 promotes proliferation and survival of colorectal cancer cells with KRAS mutations and xenograft tumor progression in mice via intracellular synthesis of aspartate. Gastroenterology. 2016;151(5):945-960.e946.

82. Tang Z, Li C, Kang B, Gao G, Li C, Zhang Z. GEPIA: a web server for cancer and normal gene expression profiling and interactive analyses. Nucleic Acids Res. Epub 2017 Apr 12.

83. Jeong J, Eide DJ. The SLC39 family of zinc transporters. Mol Aspects Med. 2013;34(2-3):612-619.

84. Eide DJ. Zinc transporters and the cellular trafficking of zinc. Biochim Biophys Acta. 2006;1763(7):711-722. 
85. Prasad AS, Beck FW, Snell DC, Kucuk O. Zinc in cancer prevention. Nutr Cancer. 2009;61(6):879-887.

86. Marshall KM, Laval M, Estacio O, et al. Activation by zinc of the human gastrin gene promoter in colon cancer cells in vitro and in vivo. Metallomics. 2015;7(10):1390-1398.

87. Hessels AM, Taylor KM, Merkx M. Monitoring cytosolic and ER $\mathrm{Zn}(2+)$ in stimulated breast cancer cells using genetically encoded FRET sensors. Metallomics. 2016;8(2):211-217.

88. Taylor KM, Vichova P, Jordan N, Hiscox S, Hendley R, Nicholson RI. ZIP7-mediated intracellular zinc transport contributes to aberrant growth factor signaling in antihormone-resistant breast cancer cells. Endocrinology. 2008;149(10):4912-4920.

89. Cohen L, Azriel-Tamir H, Arotsker N, Sekler I, Hershfinkel M. Zinc sensing receptor signaling, mediated by GPR39, reduces butyrateinduced cell death in HT29 colonocytes via upregulation of clusterin. PLoS One. 2012;7(4):e35482.

90. Rudolf E, Rudolf K. Low zinc environment induces stress signaling, senescence and mixed cell death modalities in colon cancer cells. Apoptosis. 2015;20(12):1651-1665.

91. Chandler P, Kochupurakkal BS, Alam S, Richardson AL, Soybel DI, Kelleher SL. Subtype-specific accumulation of intracellular zinc pools is associated with the malignant phenotype in breast cancer. Mol Cancer. 2016;15:2.

92. Kagara N, Tanaka N, Noguchi S, Hirano T. Zinc and its transporter ZIP10 are involved in invasive behavior of breast cancer cells. Cancer Sci. 2007;98(5):692-697.

93. Takatani-Nakase T. Migration behavior of breast cancer cells in the environment of high glucose level and the role of zinc and its transporter. Yakugaku Zasshi. 2013;133(11):1195-1199.

94. Cui X, Zhang Y, Yang J, et al. ZIP4 confers resistance to zinc deficiencyinduced apoptosis in pancreatic cancer. Cell Cycle. 2014;13(7): $1180-1186$

95. Miyai T, Hojyo S, Ikawa T, et al. Zinc transporter SLC39A10/ZIP10 facilitates antiapoptotic signaling during early B-cell development. Proc Natl Acad Sci USA. 2014;111(32):11780-11785.

96. Komori J, Marusawa H, Machimoto T, et al. Activation-induced cytidine deaminase links bile duct inflammation to human cholangiocarcinoma. Hepatology. 2008;47(3):888-896.

97. Koepsell H, Endou H. The SLC22 drug transporter family. Pflugers Arch. 2004;447(5):666-676.

98. Boyer JL. Bile formation and secretion. Compr Physiol. 2013; 3(3):1035-1078.

99. Kullak-Ublick GA, Beuers U, Paumgartner G. Hepatobiliary transport. J Hepatol. 2000;32(1 Suppl):3-18.

100. Nyquist MD, Prasad B, Mostaghel EA. Harnessing solute carrier transporters for precision oncology. Molecules. 2017;22(4).pii:E539.

101. Hu G, Henke A, Karpowicz RJ, Jr, et al. New fluorescent substrate enables quantitative and high-throughput examination of vesicular monoamine transporter 2 (VMAT2). ACS Chem Biol. 2013;8(9):1947-1954.

102. Ulanovskaya OA, Cui J, Kron SJ, Kozmin SA. A pairwise chemical genetic screen identifies new inhibitors of glucose transport. Chem Biol. 2011;18(2):222-230.

103. Lin L, Yee SW, Kim RB, Giacomini KM. SLC transporters as therapeutic targets: emerging opportunities. Nat Rev Drug Discov. 2015;14(8):543-560.

104. Andrianopoulos GD, Nelson RL, Barch DH, Nyhus LM. Sulfasalazine alters the character of dimethylhydrazine-induced colorectal carcinoma in rats. Anticancer Res. 1989;9(6):1725-1728.

105. Suzuki S, Sakamoto S, Mitamura T, Sassa S, Kudo H, Yamashita Y. Preventive effects of sulphasalazine on colorectal carcinogenesis in mice with ulcerative colitis. In Vivo. 2000;14(3):463-466.

106. Ma MZ, Chen G, Wang P, et al. Xc- inhibitor sulfasalazine sensitizes colorectal cancer to cisplatin by a GSH-dependent mechanism. Cancer Lett. 2015;368(1):88-96.
107. Huang Z, Guo KJ, Guo RX, He SG. Effects of 5-fluouracil combined with sulfasalazine on human pancreatic carcinoma cell line BxPC-3 proliferation and apoptosis in vitro. Hepatobiliary Pancreat Dis Int. 2007;6(3):312-320.

108. Muerkoster S, Arlt A, Witt M, et al. Usage of the NF-kappaB inhibitor sulfasalazine as sensitizing agent in combined chemotherapy of pancreatic cancer. Int J Cancer. 2003;104(4):469-476.

109. Tanaka T, Nishikawa A, Shima H, et al. Inhibitory effects of chlorogenic acid, reserpine, polyprenoic acid (E-5166), or coffee on hepatocarcinogenesis in rats and hamsters. Basic Life Sci. 1990;52:429-440.

110. Dunnick JK, Eustis SL. Decreases in spontaneous tumors in rats and mice after treatment with amphetamine. Toxicology. 1991;67(3):325-332.

111. Sugie S, Tanaka T, Kuniyasu T, Mori H. Inhibitory effect of reserpine on $\mathrm{N}$-2-fluorenylacetamide-induced hepatocarcinogenesis in rats. Toxicol Lett. 1987;37(2):115-119.

112. De Miglio MR, Muroni MR, Simile MM, et al. Implication of Bcl-2 family genes in basal and D-amphetamine-induced apoptosis in preneoplastic and neoplastic rat liver lesions. Hepatology. 2000;31(4):956-965.

113. van Rooijen N, Kors N, ter Hart H, Claassen E. In vitro and in vivo elimination of macrophage tumor cells using liposome-encapsulated dichloromethylene diphosphonate. Virchows Arch B Cell Pathol Incl Mol Pathol. 1988;54(4):241-245.

114. Griesmann H, Drexel C, Milosevic N, et al. Pharmacological macrophage inhibition decreases metastasis formation in a genetic model of pancreatic cancer. Gut. 2017;66(7):1278-1285.

115. Kruse J, von Bernstorff W, Evert K, et al. Macrophages promote tumour growth and liver metastasis in an orthotopic syngeneic mouse model of colon cancer. Int J Colorectal Dis. 2013;28(10):1337-1349.

116. Zhang W, Zhu XD, Sun HC, et al. Depletion of tumor-associated macrophages enhances the effect of sorafenib in metastatic liver cancer models by antimetastatic and antiangiogenic effects. Clin Cancer Res. 2010;16(13):3420-3430.

117. Gil-Ad I, Zolokov A, Lomnitski L, et al. Evaluation of the potential anti-cancer activity of the antidepressant sertraline in human colon cancer cell lines and in colorectal cancer-xenografted mice. Int $J$ Oncol. 2008;33(2):277-286.

118. Kannen V, Marini T, Turatti A, et al. Fluoxetine induces preventive and complex effects against colon cancer development in epithelial and stromal areas in rats. Toxicol Lett. 2011;204(2-3):134-140.

119. Kannen V, Hintzsche H, Zanette DL, et al. Antiproliferative effects of fluoxetine on colon cancer cells and in a colonic carcinogen mouse model. PLoS One. 2012;7(11):e50043.

120. Kannen V, Garcia SB, Silva WA, Jr, et al. Oncostatic effects of fluoxetine in experimental colon cancer models. Cell Signal. 2015;27(9): $1781-1788$

121. Kuwahara J, Yamada T, Egashira N, et al. Comparison of the anti-tumor effects of selective serotonin reuptake inhibitors as well as serotonin and norepinephrine reuptake inhibitors in human hepatocellular carcinoma cells. Biol Pharm Bull. 2015;38(9):1410-1414.

122. Mun AR, Lee SJ, Kim GB, Kang HS, Kim JS, Kim SJ. Fluoxetineinduced apoptosis in hepatocellular carcinoma cells. Anticancer Res. 2013;33(9):3691-3697.

123. Shiozaki A, Miyazaki H, Niisato N, et al. Furosemide, a blocker of Na+/ $\mathrm{K}+/ 2 \mathrm{Cl}-$ cotransporter, diminishes proliferation of poorly differentiated human gastric cancer cells by affecting G0/G1 state. $J$ Physiol Sci. 2006;56(6):401-406.

124. Malamas AS, Jin E, Zhang Q, Haaga J, Lu ZR. Anti-angiogenic effects of bumetanide revealed by DCE-MRI with a biodegradable macromolecular contrast agent in a colon cancer model. Pharm Res. 2015;32(9):3029-3043.

125. Seol HS, Lee SE, Song JS, et al. Glutamate release inhibitor, riluzole, inhibited proliferation of human hepatocellular carcinoma cells by elevated ROS production. Cancer Lett. 2016;382(2):157-165. 


\section{Publish your work in this journal}

Cancer Management and Research is an international, peer-reviewed open access journal focusing on cancer research and the optimal use of preventative and integrated treatment interventions to achieve improved outcomes, enhanced survival and quality of life for the cancer patient. The manuscript management system is completely online and includes a very quick and fair peer-review system, which is all easy to use. Visit http://www.dovepress.com/testimonials.php to read real quotes from published authors.

Submit your manuscript here: https://www.dovepress.com/cancer-management-and-research-journal 